\title{
Modelling Magnetization Transfer Considering Spin-Locking Effects
}

\author{
Claus Kiefer \\ Support Center for Advanced Neuroimaging (SCAN), Institute for Diagnostic and Interventional Neuroradiology, \\ University Hospital Inselspital and University of Bern, Bern, Switzerland \\ Email: claus.kiefer@insel.ch
}

Received 29 September 2014; revised 27 October 2014; accepted 26 November 2014

Copyright (c) 2014 by author and Scientific Research Publishing Inc.

This work is licensed under the Creative Commons Attribution International License (CC BY). http://creativecommons.org/licenses/by/4.0/

(c) () Open Access

\section{Abstract}

Purpose: Recently it was demonstrated that spin-locking (SL) effects can manifest as pseudo magnetization transfer (MT). To our best knowledge the MT models proposed so far cannot distinguish between saturation effects caused by the MT preparation pulses and SL phenomena. Therefore a new MT model is proposed. Materials and Methods: A binary spin-bath model for magnetization transfer was extended in that sense that SL effects are considered. The new modified spin bath model was tested for a phantom with different agar concentrates $(2 \%, 4 \%, 8 \%)$ and a $\mathrm{MnCl}_{2}$ (0.3 mM) solution. Results: The mean fitting error is 3.2 times lower for the modified model compared to the original model. Especially the parameter $F$ for the fractional part of the bounded proton pool describes the situation for the $\mathrm{MnCl}_{2}(\mathrm{~F}=0)$ better than the original model $(\mathrm{F}=0.004)$. Conclusion: The proposed mathematical modifications of the binary spin-bath model considering SL seem to be a step in the right direction in that sense that the effects associated with SL are not interpreted as magnetization transfer.

\section{Keywords}

MRI, Magnetization Transfer, Spin-Locking

\section{Introduction}

The magnetization transfer (MT) contrast [1]-[3] and related model parameters meanwhile deliver an important way to characterize macromolecules resp. diagnostic biomarkers in living tissue as shown in [4]. As already demonstrated by Stanisz [5], the MT ratio, which is often clinically used, is prone to T1 and T2 effects and therefore a sophisticated model based approach is more suitable. The binary spin-bath model proposed by Sled [1]-[3] is one of the methodologically most sounded techniques so far. However, as shown by Ulmer [6], this model 
should also include spin-locking (SL) effects, because the model used in [1]-[3] cannot distinguish between saturation effects caused by the MT sequences and MT preparation pulses and spin-locking. This is a problem because the model parameter values such as the one for the fraction of the macromolecules maybe wrong.

In this technical note the original spin-bath model proposed by Sled was extended in that sense that SL effects are considered. The new modified spin bath model was tested for a phantom with different agar concentrates $(2 \%, 4 \%, 8 \%)$ and a $\mathrm{MnCl}_{2}(0.3 \mathrm{mM})$ solution.

\section{Methods and Materials}

The magnetization transfer is described by a system of modified Bloch Equations (1)-(3). As this system is not analytically solvable, Sled proposed a 3-step-strategy divided in a phase of instantaneous saturation of the free pool, the free precession of the free pool and the continuous saturation of the bounded pool. This procedure enables to decouple the transversal magnetization of the free pool from the other components. We follow this approach and start with the description of the longitudinal magnetization assuming that the transversal components are zero at the end of a pulse period $M_{x, f}=M_{y, f}=0$ which can be guaranteed by relaxation or specific spoiling (indices $f$ : free, $b$ : bounded)

$$
\begin{gathered}
\mathrm{d} M_{z, f} / \mathrm{d} t=R_{1, f}\left(M_{0, f}-M_{z, f}\right)-k_{f} M_{z, f}+k_{b} M_{z, b} \\
\mathrm{~d} M_{z, b} / \mathrm{d} t=R_{1, b}\left(M_{0, b}-M_{z, b}\right)-k_{b} M_{z, b}+k_{f} M_{z, f}-W M_{z, b} \\
W=\pi \omega_{1}(t)^{2} G(\Delta) \\
G(\Delta)=T_{2, b} \int_{0}^{1} \frac{1}{\left|3 u^{2}-1\right|} \exp \left[-2\left(\frac{2 \pi \Delta T_{2, b}}{3 u^{2}-1}\right)^{2}\right] \\
\Delta / \gamma=\left(\omega_{0}-\omega_{R F}\right) / \gamma .
\end{gathered}
$$

where $R_{1, f}$ is the T1 relaxation rate (1/sec) of the free pool, $k_{f}$ and $k_{b}$ are the exchange rates $(1 / \mathrm{sec})$ of the free and bounded pool resp., $W$ (3) is the transition rate and $G$ (4) is the absorption lineshape of the bounded pool, $T_{2, b}$ is the T2 relaxation time (us) of the bounded pool and $\omega_{1}$ is the "frequency" of the continuous wave. According to Ulmer [6] the spin-locking can be described as

$$
\begin{gathered}
\mathrm{d} M_{\rho} / \mathrm{d} t=-R_{1, \rho}\left(M_{\rho}(t)-M_{\rho}(E Q)\right) \\
M_{\rho}(E Q)=\left(T_{1, \rho} / T_{1}\right) M_{0, b} \sin \theta \\
\theta=\operatorname{atan}\left((\Delta / \gamma) / B_{1}\right) \\
R_{1, \rho}=R_{1, f} \sin ^{2} \theta+R_{2, f} \cos ^{2} \theta
\end{gathered}
$$

Equation (6) has the solution

$$
M_{\rho}(t)=M_{\rho}(E Q)\left(1-\exp \left[-t / T_{1, \rho}\right]\right)
$$

In the context of the original model $(1-5)$ a significant reduction of the signal intensities for off-resonance frequencies greater than $500 \mathrm{~Hz}$ (no direct saturation) would be interpreted as magnetization transfer effects. To avoid this, the new model must consider the spin-locking (SL) related changes of the z-component in equation (2):

$$
\begin{aligned}
\mathrm{d} M_{z, b} / \mathrm{d} t & =R_{1, b}\left(M_{0, b}-M_{z, b}\right)-k_{b} M_{z, b}+k_{f} M_{z, f}-W M_{z, b}+c_{S L} \mathrm{~d} M_{\rho} / \mathrm{d} t \sin \theta \\
& =R_{1, b}\left(M_{0, b}-M_{z, b}\right)-k_{b} M_{z, b}+k_{f} M_{z, f}-W M_{z, b}+c_{S L} R_{1, f} \exp \left[-t / T_{1, \rho}\right] \sin ^{2} \theta M_{0, b}
\end{aligned}
$$

The coupling parameter $c_{S L}$ is considered either as a free adjustable degree of freedom or a constant (for comparison purposes).

In matrix form the differential equation system $(1,11)$ can be written as: 


$$
\mathrm{d} \boldsymbol{M}(t) / \mathrm{d} t=\boldsymbol{A}_{c w} \boldsymbol{M}(t)+\boldsymbol{B}(t) \boldsymbol{M}_{0}
$$

(bold type expresses matrices and vectors) where $\boldsymbol{M}(t)=\left(M_{z, f}(t), M_{z, b}(t)\right)^{\mathrm{T}}, \quad \boldsymbol{M}_{0}=\left(M_{0, f}, M_{0, b}\right)^{\mathrm{T}}$,

$$
\boldsymbol{A}_{c w}=\left[\begin{array}{cc}
-\left(R_{1, f}+k_{f}\right) & k_{b} \\
k_{f} & -\left(R_{1, b}+k_{b}+W\right)
\end{array}\right], \quad \boldsymbol{B}(t)=\left[\begin{array}{cc}
R_{1, f} & 0 \\
0 & R_{1, b}+h(t)
\end{array}\right], \quad h(t)=c_{S L} R_{1, f} \exp \left[-t / T_{1, \rho}\right] \sin ^{2} \theta
$$

The effect of both the MT pulse and the excitation pulse on the free pool is modelled as an instantaneous fractional saturation $\boldsymbol{S}$ of the longitudinal magnetization. The effect on the z-magnetization before $\boldsymbol{M}^{-}$and after the pulses $\boldsymbol{M}^{+}$can be written as

$$
\boldsymbol{M}^{+}=\left[\begin{array}{cc}
S_{f} & 0 \\
0 & S_{b}
\end{array}\right] \boldsymbol{M}^{-}=\boldsymbol{S} \boldsymbol{M}^{-}
$$

in which $S_{f}$ and $S_{b}$ denote the fractions of the longitudinal magnetization that remains after the pulses for the free and restricted pool respectively.

The general solution of (12) is

$$
\boldsymbol{M}(t)=\exp \left[\left(t-t_{0}\right) \boldsymbol{A}_{c w}\right] \boldsymbol{S} \boldsymbol{M}^{-}+\int_{t_{0}}^{t} \exp \left[(t-s) \boldsymbol{A}_{c w}\right] \boldsymbol{B}(s) \boldsymbol{M}_{0} \mathrm{~d} s
$$

From the periodicity property of the sequence $\boldsymbol{M}(T R)=\boldsymbol{M}(0)$ it follows that

$$
\boldsymbol{M}=\left[\boldsymbol{I}-\exp \left[\boldsymbol{A}_{c w} T R\right] \boldsymbol{S}\right]^{-1} \exp \left[\boldsymbol{A}_{c w} T R\right] \boldsymbol{M}_{c w}^{s s}
$$

The steady state magnetization $\boldsymbol{M}_{c w}^{s s}$ equals the magnetization due to a long period of continuous wave irradiation of the restricted pool and follows from $\mathrm{d} \boldsymbol{M}(t) / \mathrm{d} t=0$ and with (12) $\boldsymbol{M}(T R)=-\boldsymbol{A}_{c w}^{-1} \boldsymbol{B}(T R) \boldsymbol{M}_{0}$ :

$$
\boldsymbol{M}_{c w}^{s s}=\frac{1}{\operatorname{det} \boldsymbol{A}_{c w}}\left[\begin{array}{c}
\left(R_{1, b}+k_{b}+W\right) R_{1, f} M_{0, f}+k_{b}\left(R_{1, b}+h(T R)\right) M_{0, b} \\
k_{f} R_{1, f} M_{0, f}+\left(R_{1, f}+k_{f}\right)\left(R_{1, b}+h(T R)\right) M_{0, b}
\end{array}\right]
$$

With det $\boldsymbol{A}_{c w}=R_{1, f} R_{1, b}+R_{1, f} k_{b}+R_{1, f} W+k_{f} R_{1, b}+k_{f} W$.

Nonlinear fitting was performed with a Levenberg-Marquardt algorithm within a Matlab (R2014a) framework. The free fitting parameters are $\left[k_{f}, k_{b}, T_{2, b}, c_{S L}\right]$, other parameters such as $F$ and $\boldsymbol{M}_{0}$ are determined by $F=k_{f} / k_{b}, M_{0, f}=1$ and $M_{0, b}=F$. The B1 value in (8) is typically in the range of a few micro-Tesla, in our setting 60uT.

The sequence is a 2D-FLASH sequence. The sequence parameters are as follows: flip angle 540 degree (MT preparation pulse), 15 degree (excitation pulse), 30 slices, gap 0 , voxel size $=2 \mathrm{~mm}$ isotropic, 10 frequency samples, matrix size $=128, T R / T E=30 / 4.8 \mathrm{~ms}, B W=390 \mathrm{~Hz} / \mathrm{Px}$.

Imaging was performed on a 3.0Tesla whole body scanner. The phantom (Figure 1$)$ is a cylinder $(13 \mathrm{~cm} \mathrm{di-}$ ameter, $10 \mathrm{~cm}$ height), filled with water and four integrated tubes filled with agar gel (2\%, $4 \%, 8 \%)$ and manganese chloride $0.3 \mathrm{mM}$ respectively. The phantom temperature was kept constant.

\section{Results}

The mean MT-effects for Agar 8\%, 4\%, 2\%, $\mathrm{MnCl}_{2}$ according to the intensity values listed in Table 1 are

$$
(S(\Delta f=16)-S(\Delta f=1)) / S(\Delta f=16)=0.27,0.15,0.09,0.06 \text {. }
$$

In Figure 2 the fitting results are shown for an exemplary slice using the original model (Figure 2(a)) and the modified model (Figure 2(b)). If we compare the fitting results the mean error is 3.2 times greater using the original Sled model than the modified model. This result does not change if the coupling parameter $c_{S L}$ is not considered as a free adjustable degree of freedom but if it is set to a constant (e.g. 1, assuming the spin locking is generally present).

Numerical post processing according to the original model provides F values $200 \pm 2.3,100 \pm 3.3,45 \pm 5.9$, 


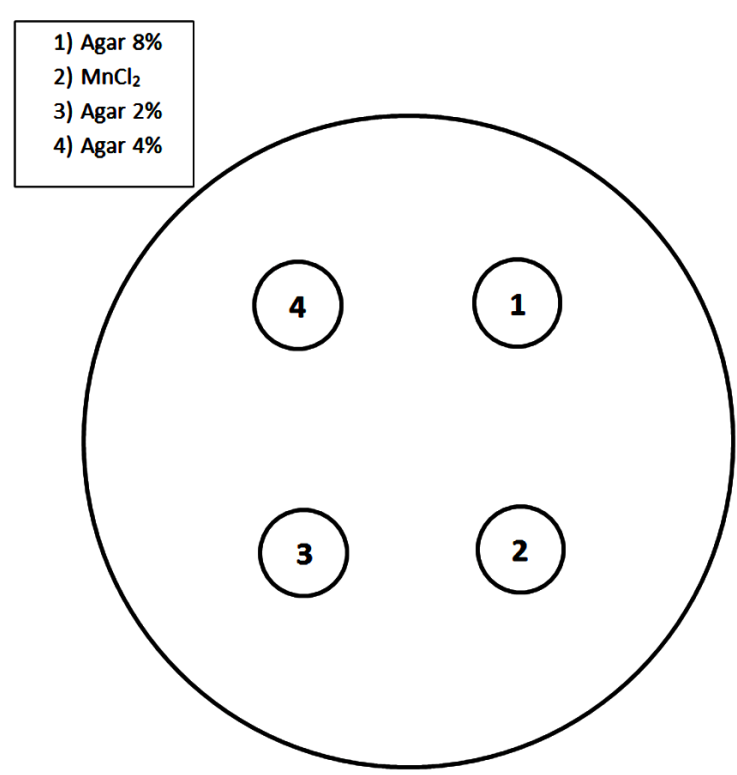

Figure 1. The phantom is a cylinder $(13 \mathrm{~cm}$ diameter, 10 $\mathrm{cm}$ height), filled with water and four integrated tubes filled with agar gel $(2 \%, 4 \%, 8 \%)$ and manganese chloride $\left(\mathrm{MnCl}_{2}\right) 0.3 \mathrm{mM}$ respectively.

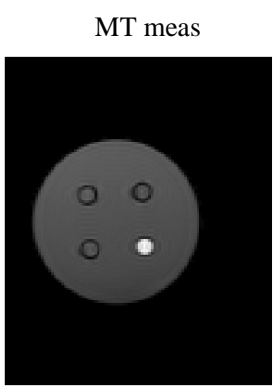

MT meas

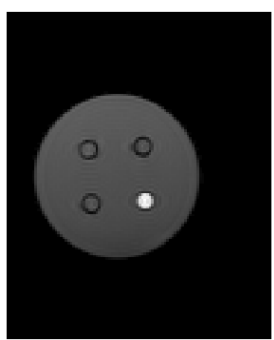

MT fit

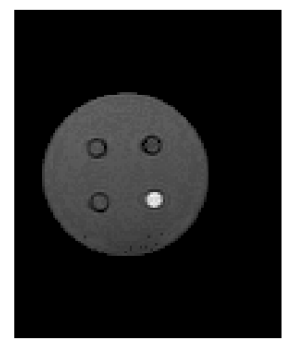

(a)

MT fit

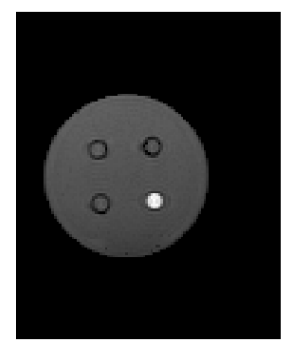

(b)

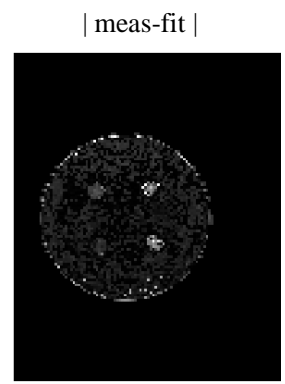

| meas-fit |

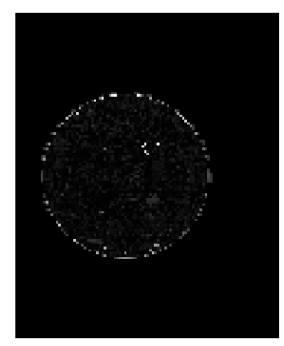

Figure 2. The fitting results are shown for an exemplary slice using the original model (2a) and the modified model (2b).

$41 \pm 3.5$ for Agar 8\%, 4\%, 2\%, $\mathrm{MnCl}_{2}$ (Figure 3(a)) and $201 \pm 2.4,100 \pm 4.0,45 \pm$ 5.1, 0 (Figure 3(b)) using the modified model (scaled with 1e5).

The non-vanishing $\mathrm{F}$ values and the significant fitting error within the $\mathrm{MnCl}_{2}$ region in the case of the original spin-bath model are present regardless of the T1 or T2 values (which were set to arbitrary values for test purposes, e.g. 1000 or $10,000 \mathrm{~ms}$ ) which means that indeed SL effects have to be considered in general (the measured T1/T2 values of $\mathrm{MnCl}_{2}$ are 140/12 ms (literature 144/14 ms)).

The values of the $T_{2, b}$ parameter for the original and modified model are very similar: $T_{2, b}($ Agar $8 \%)=12.95$ 


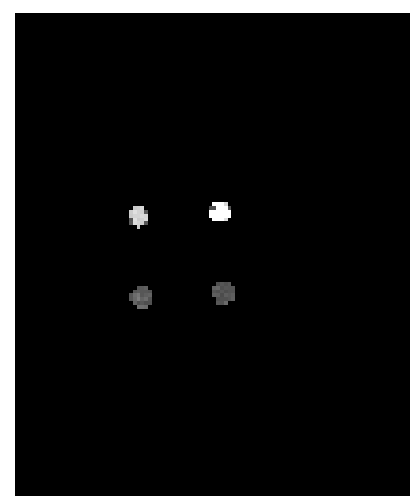

(a)

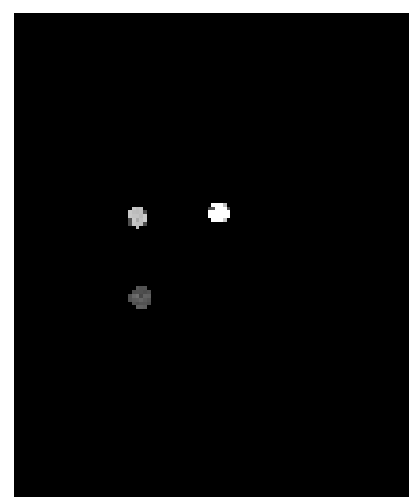

(b)

Figure 3. F maps for the original (3a) and the modified model (3b).

Table 1. Intensity values due to the MT-effects for Agar $8 \%, 4 \%, 2 \%, \mathrm{MnCl}_{2}$.

\begin{tabular}{cccccccc}
\hline Intensities & $\square \mathbf{F}=\mathbf{1 6}[\mathbf{k H z}]$ & $\mathbf{1 2}$ & $\mathbf{1 0}$ & $\mathbf{8}$ & $\mathbf{4}$ & $\mathbf{2}$ & $\mathbf{1}$ \\
\hline Agar 8\% & 184.15 & 181.14 & 180.14 & 179.14 & 174.14 & 162.13 & 135.11 \\
Agar 4\% & 201.12 & 200.12 & 199.11 & 198.12 & 196.12 & 188.11 & 170.11 \\
Agar 2\% & 182.12 & 181.13 & 181.12 & 181.12 & 180.13 & 177.13 & 166.12 \\
MnCl$_{2}$ & 507.25 & 507.26 & 506.25 & 506.26 & 504.26 & 498.26 & 479.25 \\
\hline
\end{tabular}

$\pm 2.4 \mu \mathrm{s}, T_{2, b}($ Agar $4 \%)=12.98 \pm 0.2 \mu \mathrm{s}, T_{2, b}($ Agar $2 \%)=12.98 \pm 0.5 \mu \mathrm{s}, T_{2, b}\left(\mathrm{MnCl}_{2}\right)=1 \mu \mathrm{s}$. The $T_{2, b}$ values for distilled water and $\mathrm{MnCl}_{2}$ are arbitrarily set to one because the z-spectrum in the context of the new model is just a constant, except at the resonance (zero).

The $k_{f}$ values using the original model are $k_{f}\left(\right.$ Agar 8\%) $=4.25 \pm 0.6, k_{f}\left(\right.$ Agar 4\%) $=1.66 \pm 0.6, k_{f}($ Agar $2 \%)$ $=0.56 \pm 0.1, k_{f}\left(\mathrm{MnCl}_{2}\right)=0.42 \pm 0.1$. The $k_{f}$ values using the modified model are $k_{f}($ Agar $8 \%)=1.98 \pm 0.2, k_{f}$ $($ Agar $4 \%)=1.02 \pm 0.1, k_{f}($ Agar $2 \%)=0.37 \pm 0.1, k_{f}\left(\mathrm{MnCl}_{2}\right)=0$.

\section{Conclusion}

The proposed mathematical modifications of the binary spin-bath model considering spin-locking seem to be a step in the right direction in that sense that the effects associated with spin-locking are not interpreted as magnetization transfer furthermore. This could be verified by the F parameter which is really zero within the region of $\mathrm{MnCl}_{2}$ using the modified model. The $T_{2, b}$ values of $13 \mu$ s for the agar solutions correspond very well with the literature [1]-[3]. The fact, that the good fitting result for the new model is not simply associated with a higher degree of freedom (e.g. using five instead of four free parameters) further supports the proposed approach. The recently proposed extensions on the field of sequence design [7] may further help to transfer this promising technique for the detection of macromolecules resp. biomarkers into clinical routine. 


\section{Acknowledgements}

This work was supported by the Ruth \& Arthur Scherbarth foundation, grant 2249.

\section{References}

[1] Pike, G.B. (1996) Pulsed Magnetization Transfer Contrast in Gradient Echo Imaging: A Two Pool Analytic Description of Signal Response. Magnetic Resonance in Medicine, 36, 95-103. http://dx.doi.org/10.1002/mrm.1910360117

[2] Sled, J.G. and Pike, G.B. (2000) Quantitative Interpretation of Magnetization Transfer in Spoiled Gradient Echo MRI Sequences. Journal of Magnetic Resonance, 145, 24-36. http://dx.doi.org/10.1006/jmre.2000.2059

[3] Sled, J.G. and Pike, G.B. (2001) Quantitative Imaging of Magnetization Transfer Exchange and Relaxation Properties in Vivo Using MRI. Magnetic Resonance in Medicine, 46, 923-931.

[4] Kiefer, C., Brockhaus, L., Cattapan-Ludewig, K., Ballinari, P., Burren, Y., Schroth, G. and Wiest, R. (2009) Multiparametric Classification of Alzheimer's Disease and Mild Cognitive Impairment: The Impact of Quantitative Magnetization Transfer MR Imaging. NeuroImage, 48, 657-667.

[5] Stanisz, G.J., Yoon, R.S., Joy, M.L.G. and Henkelman, R.M. (2002) Why Does MTR Change with Neuronal Depolarization? Magnetic Resonance in Medicine, 47, 472-475. http://dx.doi.org/10.1002/mrm.10071

[6] Ulmer, J.L., Mathews, V.P., Hamilton, C.A., Elster, A.D. and Moran, P.R. (1996) Magnetization Transfer or SpinLock? An Investigation of Off-Resonance Saturation Pulse Imaging with Varying Frequency Offsets. AJNR-American Journal of Neuroradiology, 17, 805-819.

[7] Kiefer, C. (2014) Simultaneous Echo Refocused Magnetization Transfer Imaging. Open Journal of Medical Imaging, 4, 154-158. http://dx.doi.org/10.4236/ojmi.2014.43021 
Scientific Research Publishing (SCIRP) is one of the largest Open Access journal publishers. It is currently publishing more than 200 open access, online, peer-reviewed journals covering a wide range of academic disciplines. SCIRP serves the worldwide academic communities and contributes to the progress and application of science with its publication.

Other selected journals from SCIRP are listed as below. Submit your manuscript to us via either submit@scirp.org or Online Submission Portal.
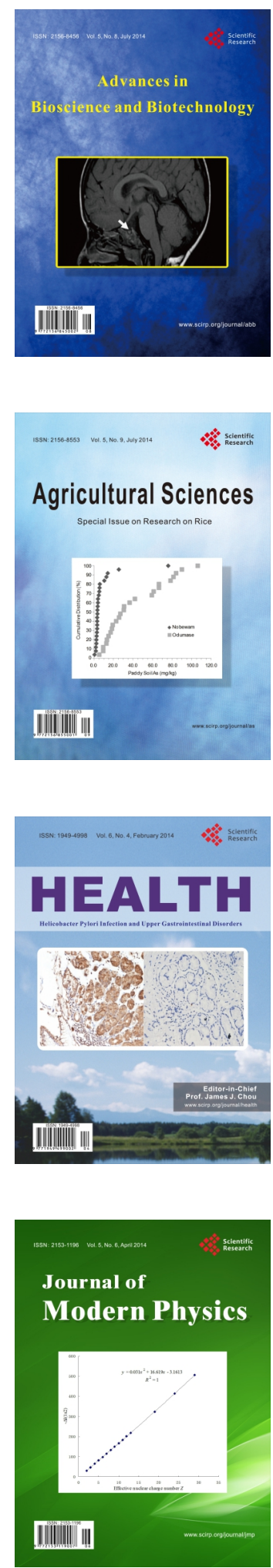
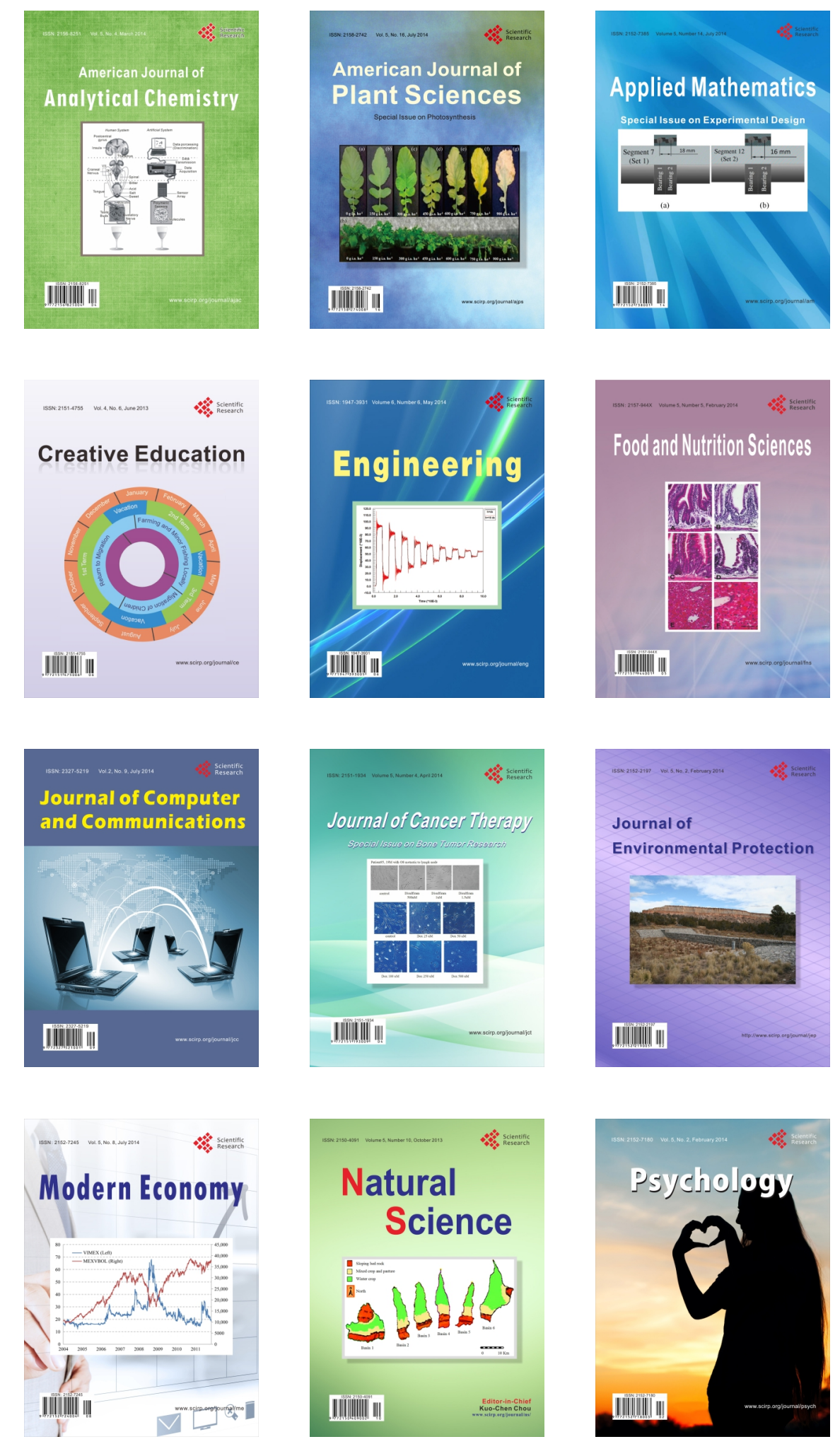